\title{
Diabetes mellitus y lesiones del pie*
}

\author{
Salvador López-Antuñano, M.D., (1) \\ Francisco J. López-Antuñano, M.D., M.P.H.(2)
}

E n la Declaración de las Américas sobre Diabetes - Mellitus, ${ }^{1,2}$ se estima que actualmente hay en el mundo alderredor de 135 millones de diabéticos y se espera que esta cifra se eleve a 300 millones en los próximos 25 años; el aumento será de $40 \%$ en los países desarrollados y de $70 \%$ en los países en vías de desarrollo. El 25\% del total de casos se concentra en los países del nuevo mundo, y la tendencia indica que para el año 2000 habrá 45 millones de diabéticos en esa región. Los 10 países con mayor número de personas con diabetes mellitus (DM) son la India (19 millones), China (16 millones), Estados Unidos de América (13.9 millones), Federación Rusa (8.9 millones), Japón (6.3 millones), Brasil (4.9 millones), Indonesia (4.5 millones), Pakistan (4.3 millones), México (3.8 millones) y Ukrania (3.6 millones). Sus repercusiones en el mundo en términos de pérdida de días ajustados a años de vida saludables se calcularon en 11103000 en 1990, y se estima que esta cifra será de 10805000 para el año 2020.

La DM se ha asociado a una multiplicidad de condiciones como las ambientales o la edad, entre otras; por ejemplo, la prevalencia de esta enfermedad aumenta particularmente en grupos sociales que han mudado rápidamente del estilo de vida tradicional al moderno; por otro lado, la DM tipo 1 es la que prevalece entre los niños del planeta.,

En Estados Unidos de América (EUA) 90\% de los diabéticos son mayores de 50 años; $20 \%$ se hospitaliza por las complicaciones que produce la DM en el pie, las cuales se manifiestan después de los 50 años de edad; una tercera parte presenta enfermedad vascular periférica, y $7 \%$ requiere cirugía vascular o amputación.
La mortalidad aumenta en diabéticos mayores de 45 años en comparación con enfermos de menor edad. ${ }^{5}$

En México, la distribución de la mortalidad por DM parece tener un comportamiento desigual en el ámbito nacional. La tasa de mortalidad ajustada por edad es de 56.41 en Oaxaca, mientras que en Chihuahua llega a 199.85 por $10^{5}$ habitantes, por lo que el riesgo de morir por DM es 3.5 veces mayor en Chihuahua que en Oaxaca. ${ }^{6}$

No obstante que se conocen las limitaciones existentes para el diagnóstico oportuno de la DM, así como la vigilancia epidemiológica inadecuada y la ausencia de programas eficaces de prevención y control, llama la atención la creciente morbilidad y mortalidad por esta enfemedad, la cual requiere de tratamiento médico e, incluso, quirúrgico cuando las complicaciones de la misma exigen la amputación de los miembros inferiores.

\section{Definición}

La diabetes mellitus es una enfermedad metabólica crónica y compleja que se caracteriza por deficiencia absoluta o relativa de insulina, hiperglicemia crónica y otras alteraciones del metabolismo de los carbohidratos y de los lípidos; ello a su vez puede originar múltiples complicaciones microvasculares en los ojos, el riñón y las extremidades inferiores, así como neuropatías periféricas y, frecuentemente, lesiones macrovasculares y coronarias.

El pie diabético, en particular, se define como la infección, la ulceración y la destrucción de los tejidos profundos, asociadas con anormalidades neurológi-

(1) Profesor de Traumatología y 0 rtopedia. Facultad de Medicina, UN AM. Asesor, Hospital Juárez de México, Secretaría de Salud, México, D.F.

(2) Investigador Titular A. Centro de Investigación en Salud Poblacional. Instituto N acional de Salud Pública, Cuernavaca, Morelos, México. 
cas (pérdida de la sensibilidad al dolor) y vasculopatía periférica de diversa gravedad en las extremidades inferiores.? $^{\text {P }}$

El pie es particularmente vulnerable a daños circulatorios y neurológicos, y el menor trauma puede causar úlceras o infecciones. La enfermedad macrovascular de las extremidades inferiores es más común y progresa más rápidamente en presencia de la DM; tiene, asimismo, una distribución peritibial característica, a la cual se agrega la frugalidad de las arterias del pie. Así, el pie diabético se hace más vulnerable a las heridas. La alteración en la perfusión de la sangre en las extremidades inferiores tiene la mayor importancia porque da la alarma para que se adopte un método efectivo hacia la revascularización. ${ }^{8,9}$

\section{Clasificación de la DM}

Recientemente algunos investigadores ${ }^{10-13}$ se han propuesto clasificar la DM con los siguientes criterios:

1. La presencia de síntomas de DM, más un nivel de glucosa en plasma casual de $11.1 \mathrm{mmol} / 1$ (200 $\mathrm{mg} / \mathrm{dl})$.

2. Un nivel de glucosa en plasma de $7.0 \mathrm{mmol} / 1$ $(126 \mathrm{mg} / \mathrm{dl})$ cuando el paciente está en ayunas.

3. Un nivel de dos horas con $11.1 \mathrm{mmol} / 1$ durante la prueba oral de tolerancia a la glucosa, administrando al paciente el equivalente a $75 \mathrm{~g}$ de glucosa anhidra disuelta en agua.

Asimismo, proponen la confirmación del diagnóstico mediante una nueva observación de alguna de las anormalidades anteriores, repitiendo las tres pruebas en días diferentes.

Para estudios poblacionales, se recomienda el nivel de $7.0 \mathrm{mmol} / 1$ de glucosa en plasma, en ayunas, como medida única para definir la DM.

La DM tipo 1 se refiere a pacientes cuyo problema metabólico es la deficiencia absoluta de insulina. En la DM tipo 2 (al que pertenece la gran mayoría de los pacientes), la insulina está presente, pero su cantidad no responde al aumento en su demanda (resistencia a la insulina). Así, los términos DM insulino-dependiente y DM no insulino-dependiente ya no se consideran aceptables.

Para asociar los valores de azúcar con el riesgo de complicaciones micro y macrovasculares, es tan útil usar directamente los datos prospectivos de glucosa en la población, como utilizar indirectamente la medida más estable de hemoglobina glucosilada (hemoglobina $_{1 c}$ ). Esta última provee de la medida "integral" o acumulada a lo largo del tiempo y evita los proble- mas diferenciales en las muestras de sangre. La confianza en el nivel de glucosa en ayunas como signo de DM compite con la confianza en el nivel de glucosa posprandial. Este último es más sensible a la presencia de la DM, ya que una pequeña disminución de la reserva secretoria malogra la habilidad de manejar la carga de glucosa, mientras que la capacidad secretoria de la insulina en ayunas es la última en desaparecer. ${ }^{14}$

Cerca de $85 \%$ de los casos de DM en países desarrollados son tipo 2 y muchos exhiben hiperinsulinemia asociada a dislipoproteinemia, obesidad, hiperuricemia y presión arterial alta.

Los estudios de las enfermedades producidas por mutantes del $\mathrm{ADN}$ mitocondrial sugieren que varios procesos degenerativos pueden estar asociados con defectos en la fosforilación oxidativa (OXPHOS). La aplicación de esta hipótesis ha permitido avanzar en el planteamiento de problemas clínicos como la enfermedad isquémica del corazón y de las arterias, la diabetes y otros..$^{15}$ La hiperglucemia por fibrosis del páncreas parece transmitirse con el carácter autosómico recesivo, y la hiperglucemia asociada a distrofia miotónica, con el carácter dominante. Los datos disponibles en relación con la hipótesis de que la variación de la frecuencia en la población de los alelos HLA-DQ es un determinante primario de la incidencia de DM tipo 1, muestran variaciones significativas en la frecuencia de los alelos DQA1 y DQB1 entre los casos de DM y los controles. En 16 países, con excepción de Japón, DQA1* Arg-52 y DQB1*no-Asp-57 (ND) fueron marcadores consistentes e independientes de la susceptibilidad a la DM tipo $1 .{ }^{16}$

El aminoácido 57 de la cadena DQb se asocia fuertemente con la susceptiblidad a la DM tipo 1 y a inmunodeficiencias humorales. ${ }^{17}$ Con base en los estudios de una necropsia en un niño con diabetes, cetoacidosis e infiltración linfocítica alderredor de los islotes de Langherhans, desde 1940 se acepta la lesión específica de la diabetes tipo 1 como un fenómeno de autoinmunidad. A partir de 1974 se ha confirmado la asociación de la DM tipo 1 con otras enfermedades autoinmunes como la de Hashimoto, Graves, Addison, ooforitis, orquitis, hipoparatiroidismo, hipofisitis y otras no hormonales como la artritis reumatoide, miastenia gravis, púrpura trombocitopénica y hepatitis crónica activa; además, en algunas de ellas, se ha demostrado por inmunofluorescencia la presencia de anticuerpos específicos contra células pancreáticas en secciones de páncreas.

Se han utilizado modelos matemáticos (MM) para calcular la oportunidad relativa (OR) a la asociación entre el polimorfismo en la región HLA-DQ y la 
DM tipo 1. El MM más compatible con las hipótesis actuales en el proceso patológico de la DM usa la clasificación de heterodímeros $\mathrm{a} / \mathrm{b}$ como factor de susceptibilidad. Una vez que se cuenta con la OR se calcula una aproximación de la prevalencia de DM tipo 1, de acuerdo con el polimorfismo en la región HLA-DQ. ${ }^{18}$

Hipotéticamente, la DM tipo 1 se ha considerado como consecuencia de una o más infecciones virales (rubéola, varicela, hepatitis, mononucleosis, citomegalovirus), las que al producir pancreatitis (insulinitis) destruyen las células $\beta$ de los islotes pancreáticos; de este modo, se reduce la producción de insulina y, consecuentemente, se produce la hiperglucemia.

\section{Fisiopatología del síndrome del pie diabético}

El pie es una maravilla biomecánica que consta de 29 articulaciones (8 mayores), 26 huesos y 42 músculos para realizar coordinada y armónicamente sus funciones básicas de movimiento, soporte, marcha y equilibrio. ${ }^{*}$ La piel plantar posee un estrato córneo (queratinoso) que responde normalmente a las demandas de fuerza, estrés, marcha, peso corporal y ejercicio. En las áreas de mayor presión aumenta su queratinización, formando callosidades, las cuales fácilmente se fragmentan y ulceran, aun en personas no diabéticas de mediana edad.

Al pie diabético se agregan problemas potenciales de los huesos y articulaciones en virtud de la insuficiencia vascular periférica y la neuropatía. El control de la glicemia parece prevenir el desarrollo de microangiopatías de la retina y el riñón y probablemente retarda la evolución de neuropatías, pero no parece tener suficiente efecto sobre la macroangiopatía. La insuficiencia vascular periférica conduce a la necrosis y la amputación. Muchas lesiones del pie diabético se inician con síntomas de neuropatía y, si aumentan las parestesias y disminuye la sensación de dolor, los traumatismos subsecuentes y repetidos producen lesiones mayores que, asociadas a la insuficiencia vascular, impiden la cicatrización. La neuropatía puede ocasionar degeneración articular (neuroartropatía de Charcot) y dar por resultado nuevos apoyos del pie que reciclan el problema de úlcera-infección.

\footnotetext{
* Los pies están dotados de puntos equivalentes al resto del organismo de los seres humanos y, como un microcosmos, nos mantienen en contacto con nuestra Madre Tierra. Los pies son la corona del Arbol de la Vida, que tiene sus raíces en el Cielo (Pía, 1997. Comunicación personal).
}

La insuficiencia vascular periférica en la DM afecta vasos sanguíneos pequeños y grandes. La macroangiopatía de la DM no es muy diferente a la de enfermos no diabéticos. Los cambios en las capas media e íntima de la pared del vaso se generan, en ambos casos, por depósitos de lípidos, colesterol y calcio, pero éstos se acumulan en mayor cantidad en los diabéticos. Además, son más extensos entre los pequeños vasos arteriales situados por debajo de la rodilla. La oclusión de estas pequeñas arterias explica la localización de las áreas de necrosis en los diabéticos. La angiopatía de vasos de mayor calibre no progresa al mismo tiempo que la de pequeños vasos. Los pequeños vasos de los ortejos exhiben arterioesclerosis más avanzada que los vasos proximales, por lo que aun en presencia de pulso pedio o tibial, el ortejo puede tener signos de insuficiencia vascular.

Cacciatori y colaboradores ${ }^{19}$ compararon la función de los nervios periféricos simpático-adrenérgicos y colinérgicos en pacientes con DM tipo 2 y con diferentes úlceras neuropáticas del pie. Los parámetros usados fueron: el reflejo postural de vasoconstricción arteriolar (RPVA) y la respuesta simpática de la piel (RSP). Ninguno de los pacientes estudiados $(n=47)$ presentó enfermedad vascular periférica clínicamente. Los resultados indicaron que las fibras nerviosas periféricas simpático-adrenérgicas y colinérgicas tienen alteraciones simultáneas tempranas en pacientes diabéticos, aun cuando no sea evidente la neuropatía clínicamente. También se mostró abolición completa de la actividad periférica simpática en este tipo de pacientes.

Con objeto de conocer: a) el efecto de la diabetes en la isquemia de la pierna, y b) el valor agregado en la apreciación de la severidad de la enfermedad isquémica, Ubbink y colaboradores ${ }^{20}$ estudiaron la microcirculación en la piel de pacientes no diabéticos y diabéticos con isquemia de la parte inferior de la pierna. Este estudio les permitió clasificar a los pacientes isquémicos como sigue: (i) asintomáticos, (ii) claudicantes, (iii) críticamente isquémicos con tensión vascular del tobillo $(<51 \mathrm{mmHg})$ o del primer ortejo $(<31$ $\mathrm{mmHg}$ ) y (iv) pacientes en los que la tensión vascular del tobillo y del primer ortejo no pudieron evaluarse debido a la esclerosis de la pared de los vasos o a las úlceras de la piel.

La combinación de la neuropatía sensorial y la isquemia tiene efecto directo adverso sobre los mecanismos de defensa del huésped; en particular hace a los pacientes con DM más vulnerables a las infecciones del pie. ${ }^{21}$ La neuropatía puede conducir a la amputación por varios caminos, los cuales incluyen la pér- 
dida de la función autonómica, sensitiva y motora de los nervios periféricos.

En el cuadro I presentamos un esquema simplificado de la fisiopatología del pie diabético y sus consecuencias.

\section{Diagnóstico}

Insuficiencia vascular periférica

Los signos y síntomas que en la exploración física deben valorarse para el diagnóstico de la insuficiencia vascular periférica en el pie diabético son: claudicación intermitente, pie frío, dolor en reposo (por bloqueo arterial e isquemia de los nervios y neuritis), mejoría con actividad y gravedad de la extremidad, pulsos ausentes, palidez a la elevación, retardo en el vaciamiento venoso al elevar la extremidad, rubor al colgar la pierna, atrofia de grasa subcutánea, piel adelgazada, pérdida de vellos del pie y pierna, uñas quebradizas y gangrena-necrosis en botón. Puede ocurrir oclusión progresiva o aguda por trombosis, produciendo dolor, edema cerúleo, parestesia, calambres, parálisis con debilidad súbita y enfriamiento del pie. En este caso el uso del equipo Doppler para medir la velocidad y la presión arterial es una práctica recomendable.

Otro de los síntomas que se deben tomar en cuenta es que la piel se torna seca -pues la sudoración disminuye- y frágil, lo que produce grietas y cuarteaduras que fácilmente se infectan. La microangiopatía de la piel (dermopatía) o granuloma anular localizado es un marcador de DM. Los procesos responsables de los cambios en los vasos cutáneos son la fragmentación de la capa basal, la laminación del material homogéneo y la aparición de tejido conectivo en la periferia de los vasos. Esto es una expresión de envejecimiento y se usa como marcador de DM en personas mayores de 50 años, pero no se explica como reacción secundaria a la disfunción de las células de los islotes del páncreas ni como resultante del metabolismo alterado de carbohidratos, máxime que la nefropatía y la retinopatía se encuentran en el comienzo de las manifestaciones de la DM. En cambio, las alteraciones en la membrana basal de los capilares se detectan un año y medio después del diagnóstico de la enfermedad. No existe demostración de que los cambios microvasculares sean causa de las complicaciones de la DM, y su relación con la polineuritis es incierta. ${ }^{5}$

\section{Isquemia}

Para evaluar adecuadamente a los pacientes con enfermedad arterial crónica oclusiva es necesario tomar en cuenta y documentar varios factores, entre los cuales está la DM. En presencia de DM o si no se puede medir la presión sanguínea periférica, la microscopía capilar, la oximetría transcutánea y la flujometría láser Doppler de ultrasonido son técnicas útiles para evaluar la microcirculación en la piel y la gravedad de la isquemia en la parte inferior de la pierna. La DM parece cambiar la perfusión microcirculatoria, especialmente en pacientes con isquemia crítica. Con la medida de la tensión de oxígeno en la piel se obtiene un valor predictivo positivo de isquemia crítica de hasta $77 \%$ en la detección, cuando se aplica un punto de corte de 30 mmHg. El 70\% de los pacientes en los que la gravedad de la isquemia no puede determinarse por medio de la medida de la tensión arterial, pueden ser clasificados como individuos con isquemia crítica, con base en la investigación de la microcirculación. La influencia de la diabetes en la microcirculación está excedida por los efectos de la ateroesclerosis cuando la enfermedad

Cuadro I

Fisiopatología del SíndRome del PIE diabético

\begin{tabular}{|c|c|c|c|c|c|}
\hline \multicolumn{6}{|c|}{ Diabetes mellitus } \\
\hline \multicolumn{3}{|c|}{ Angiopatía } & \multicolumn{3}{|c|}{ Neuropatía } \\
\hline $\begin{array}{c}\text { Macroangio patía } \\
\text { Vasos mayores } \\
\downarrow\end{array}$ & $\begin{array}{c}\text { Microangio patía } \\
\text { Arteriolas } \\
\qquad \downarrow\end{array}$ & $\begin{array}{c}\text { Microangio patía } \\
\text { Capilares } \\
\qquad \downarrow\end{array}$ & $\begin{array}{c}\text { Sistema autónomo } \\
\downarrow \\
\text { Anhidrosis } \\
\downarrow\end{array}$ & $\begin{array}{c}\text { Sistema sensitivo } \\
\downarrow \\
\text { Indoloro } \\
\downarrow\end{array}$ & $\begin{array}{l}\text { Sistema motor } \\
\quad \downarrow \\
\text { Atrofia muscular } \\
\quad \downarrow\end{array}$ \\
\hline $\begin{array}{c}\text { Trombosis } \\
\text { O clusión } \\
\downarrow \\
\text { Necrosis extensa } \\
\downarrow\end{array}$ & $\begin{array}{c}\text { Necrosis parcelar } \\
\downarrow\end{array}$ & $\begin{array}{l}\text { Atrofia de piel } \\
\text { Ulcera } \\
\qquad \downarrow\end{array}$ & $\begin{array}{c}\text { Piel seca } \\
\text { Fisuras } \\
\downarrow \\
\text { Infección } \\
\downarrow\end{array}$ & $\begin{array}{c}\text { Neuroartropatía } \\
\downarrow \\
\text { Pie deforme } \\
\downarrow \\
\text { Infección } \\
\downarrow\end{array}$ & Cambios en la carga \\
\hline \multicolumn{4}{|c|}{ Zonas de necrosis } & Gangrena & \\
\hline Amputación & Amputación menor & Amputación & Amputación & Amputación & \\
\hline
\end{tabular}


vascular se torna grave. Cuando se aplica una intervención quirúrgica o endovascular, el análisis debe incluir el estado sintomático del paciente, la evidencia anatómica patente del proceso y de la enfermedad distal y proximal, el resultado hemodinámico, la posibilidad de preservar la pierna, y la letalidad. Todos estos factores proporcionarán un mejor conocimiento del cuadro global de la enfermedad e información para poderla tratar adecuadamente. ${ }^{22}$

\section{Neuropatía}

Los signos y síntomas de la neuropatía del pie diabético son: parestesia, hiperestesia, hipoestesia, dolor radicular, pérdida de reflejos osteotendinosos, pérdida de la sensación vibratoria y de posición, anhidrosis, formación de callos en puntos de presión, úlceras tróficas, infección, cambios de talla-deformación del pie-plano-valgo-varo, desmineralización, osteólisis y articulación de Charcot. La neuropatía es frecuentemente bilateral. Las parestesias, a veces paroxísticas, se refieren como dolor o como una sensación confusa de ardor o quemadura.

El diagnóstico diferencial del pie diabético debe hacerse cuando hay presencia de alcoholismo, hernia de disco, intoxicación por metales y drogas, colagenosis, anemia perniciosa, neoplasias, uremia y lepra, entre otros padecimientos. La alteración neuropática más importante en el pie diabético es la pérdida de la sensibilidad, por lo que el pie queda expuesto a traumatismos indoloros mecánicos, químicos o térmicos. Es muy frecuente que los problemas del pie diabético inicien a causa de la "cirugía doméstica", es decir, cuando el paciente se recorta las uñas y los callos hasta planos muy profundos porque ya no tiene sensibilidad en esa región, lo que lo predispone más fácilmente a adquirir la infección. El diabético puede empezar a provocarse lesiones o a sentir manifestaciones de ellas, al intentar calentar los pies con agua caliente o cojines eléctricos, como respuesta a la sensación de pie frío. Por otro lado el uso incorrecto de un zapato que obliga al apoyo plantar defectuoso, produce lesiones traumáticas, sobre todo si la neuropatía ya produjo neuroartropatías; la consecuente desviación del eje de los huesos deforma el pie, aumenta su diámetro y lo ensancha. Con la utilización del mismo calzado agresor, el cual es tolerado por la insensibilidad, se inicia un círculo vicioso. La situación se agrava con edema, inflamación e infección a veces dolorosa. La posición deformada de los dedos es común: dedos en garra o gatillo, sobre todo si los músculos intrínsecos del pie se han afectado; a su vez estas deformaciones producen nuevas callosidades.
La neuropatía hiperglucémica diabética incluye síntomas sensoriales menores, reducción de la velocidad de conducción nerviosa y resistencia a la falla de conducción isquémica, y la hipoxia nerviosa parece tener un papel significante en su origen. Entre los fenómenos más persistentes, se encuentra la polineuropatía simétrica distal, la cual afecta predominantemente la función sensorial y autónoma. La axonopatía distal podría ser la patogenia básica. Otros fenómenos persistentes son las lesiones focales y multifocales que ocasionan neuropatías craneales, tóraco-abdominales y de la pierna, inclusive neuropatías motoras de la pierna baja (amiotrofia diabética). Algunas de éstas pueden tener base isquémica. En particular, las lesiones multifocales proximales pueden sumarse y producir una neuropatía difusa distal simétrica. Las lesiones focales, por su parte, en sitios de compresión interna o externa, pueden reflejar una susceptibilidad anormal del nervio diabético al daño por compresión. Las lesiones inflamatorias vasculares focales pueden involucrar neuropatías proximales de la pierna baja. Por último, puede ocurrir polineuropatía inflamatoria desmielinizante.

Para evaluar el tratamiento es esencial clasificar el tipo al que pertenecen los casos. Las manifestaciones que caen en la categoría de neuropatía hiperglucémica diabética no deben contaminar la evaluación de la polineuropatía distal simétrica. También se ha propuesto la evaluación seriada por biopsia de nervios. ${ }^{23}$

\section{Infección de la piel en el pie diabético}

La infección de las úlceras del pie diabético llevan al cataclismo final de eventos como la gangrena y la amputación. Esta alteración es más difícil de diagnosticar y tratar en el diabético porque la glucemia elevada interfiere con la respuesta inmune humoral y celular y dificulta o impide el transporte de antitoxinas, citocinas y antibióticos.

La infección en el pie diabético es generalmente polimicrobiana con bacterias aeróbicas de los géneros Staphylococcus y Streptococcus y, frecuentemente, con anaerobios como Bacteroides. En el diabético con infección hay predisposición a la formación de microtrombos, los cuales producen cambios isquémicos que aumentan la necrosis y la gangrena. En el no diabético la infección produce casi siempre aumento en el flujo sanguíneo con eritema y calor.

El pie diabético, con circulación pobre y piel atrófica e insensible, es extraordinariamente sensible a la infección. La presencia de microrganismos patógenos en los pliegues de los pies, las condiciones de humedad, y la proximidad con zapatos y suelo contaminados 
deben tomarse en cuenta en el insistente cuidado y la profilaxis de las infecciones en la piel y las uñas del pie diabético; asimismo, deben tomarse medidas que favorezcan la circulación; por ejemplo, los diabéticos en cama deben movilizar el talón y evitar apoyos permanentes que favorezcan úlceras de decúbito.

\section{Osteomielitis}

La detección de tejido óseo en la base de una úlcera mediante una sonda esterilizada puede ser particularmente útil en el reconocimiento de osteomielitis. Las bacterias Gram-positivas no causan infecciones que pongan en peligro la pierna; por lo general las infecciones graves son polimicrobianas. ${ }^{21}$ Craig y colaboradores $^{24}$ evaluaron la eficacia de las imágenes por resonancia magnética (RM) para el diagnóstico de la ostiomielitis en el pie diabético, estimando la correlación con los resultados de estudios anatomohistológicos de los tejidos disecados, como referencia estándar. En pacientes con diagnóstico presuntivo de osteomielitis se hicieron exámenes de RM antes de la cirugía y se examinaron los huesos. La máxima intensidad en la inversión-recuperación de imágenes de los huesos se debió a osteomielitis (sensibilidad 90\%; especificidad, $71 \%$ ). La intensidad de la señal en el caso del edema de la médula ósea se confunde con la de la osteomielitis, pero es menor. A pesar de ello, la imagen RM es útil para planear la cirugía del pie diabético, en virtud de que permite distinguir entre las áreas normales y anormales.

Harvey y Cohen ${ }^{25}$ estudiaron leucocitos marcados con Hexamethylpropylamineoxina (HMPAO), para examinar la utilidad del procedimiento de la escintigrafía en el diagnóstico de la infección de los huesos en pacientes con úlceras crónicas no cicatrizantes del pie. Los resultados escintigráficos se compararon con análisis histológico, cultivo de huesos y radiografías. En el área de supuesta infección, 40\% de los pacientes mostraron imagen positiva con acumulación focal de leucocitos (sensibilidad 86\%, especificidad 90\%). El otro $61 \%$ de los pacientes fue examinado mediante la escintigrafía trifásica con metileno difosfato (MDP), y la sensibilidad ascendió a 91\%, pero la especificidad fue mucho menor $(40 \%)$.

\section{Inflamacion/infección}

La inmunoglobulina G inespecífica marcada con 111InIgG es uno de los nuevos agentes sugeridos para la evaluación escintigráfica de la inflamación y la infección. En un estudio se evaluó la utilidad de este agente en la práctica clínica rutinaria, administrando por vía intravenosa $2 \mathrm{mg}$ IgG marcada con 75 MBq 111In (MacroScint), en posibles focos de infección o inflamación. Se hizo una imagen a las 4, 24 y 48 horas postinyección. Los resultados fueron verificados por cultivo de material, ya sea obtenido quirúgicamente o por punción, y por medio del seguimiento clínico-radiológico. Este último se hizo especialmente con los pacientes que habían tenido en su mayoría resultados negativos, inclusive escintigrafía 111In-IgG negativa. Se detectaron todas las caderas infectadas y las artropatías de rodilla, así como osteomielitis focal, infecciones del pie diabético, artritis séptica e infecciones de los tejidos. Solamente un paciente con espondilodiscitis temprana benigna fue falso negativo. Como la escintigrafía con 111In-IgG no discrimina entre la inflamación infecciosa y la estéril, debe hacerse una interpretación cuidadosa en las artrosis de cadera no cementadas, en la lesión del cuello del fémur, en recientes fracturas y en pseudoartrosis, donde la lesión puede haber sido causada por inflamación estéril y no por infección. La escintirafía con Indium-111-IgG es un instrumento muy sensible para la detección de huesos infectados y de enfermedad de las articulaciones. Si se obtienen los patrones característicos de inflamación estéril, la infección puede descartarse con un alto grado de certeza. ${ }^{26}$

\section{Estrés al frío}

Chen y colaboradores ${ }^{27}$ evaluaron la respuesta microvascular periférica a la prueba de estrés al frío aislado en pacientes con diabetes de gravedad variable. Se estudiaron cuatro grupos de diabéticos: a) con neuropatía; b) con neuropatía motosensorial periférica; c) con enfermedad vascular periférica, y d) controles. Las temperaturas digitales de los cuatro grupos disminuyó durante los 20 minutos del periodo de enfriamiento, aunque fue mayor en el grupo control; después aumentó durante los 20 minutos del periodo de recalentamiento, sin diferencia entre los grupos. La temperatura digital de los diabéticos con y sin neuropatía fue mayor que en los diabéticos con enfermedad vascular y los controles. Se notó mayor temperatura digital promedio en la piel de los pacientes de los grupos a y $b$. En los grupos a, b y c, se encontraron disminuciones significantes en los valores de la flujometría láser Doppler de ultrasonido, desde el último minuto de climatización, hasta el último minuto de la sesión de enfriamiento. Sólo en el grupo a, se observó un aumento significativo durante el recalentamiento. El aumento en el flujo digital vascular fue consistente con el puente 
arteriovenoso y el control vascular anormal en los pacientes diabéticos con y sin neuropatía. Los pacientes diabéticos con enfermedad vascular periférica tuvieron el menor valor y la menor fluctuación en la flujometría de ultrasonido, pero su temperatura digital en la piel no mostró diferencia significativa de la de las personas normales.

\section{Percepción vibratoria y discriminación térmica}

DeNeeling y colaboradores ${ }^{28}$ determinaron los niveles de reproducibilidad de la prueba-reprueba de la percepción vibratoria (PPV) y de discriminación térmica (PDT) en el pie de adultos mayores, para lo cual examinaron a 20 personas de 50-76 años de edad con DM tipo 2 y a 19 más sin DM. Se obtuvieron valores ajustados de referencia para ambos niveles analizando las relaciones entre la edad, la estatura y el sexo en 216 personas de la misma edad con tolerancia normal a la glucosa.

La PPV parece ser más reproducible que la PDT (coeficiente de exactitud 0.89 vs 0.54 ). La reproducibilidad de la PPV es inversamente proporcional a la edad y mejor para hombres que para mujeres; estas últimas tienen mayor percepción vibratoria. La diabetes y el nivel glucémico no afectan la reproducibilidad de ninguna de las pruebas; ambas están relacionadas con la edad y la estatura.

\section{Estenosis arterial}

Un estudio epidemiológico hecho por Vogt y colaboradores $^{29}$ tuvo el propósito de identificar la correlación del riesgo de enfermar o morir asociado con la presencia de estenosis de segmentos de la arteria mayor de la pierna en hombres y mujeres, evaluados en un laboratorio vascular periférico. El análisis multivariado indicó que el tabaquismo y la presión sanguínea sistólica elevada eran los factores de riesgo clave, asociados a la enfermedad arterial aislada aortoilíaca y femoropoplítea, tanto en hombres como en mujeres. En la muestra de población, la historia de DM estuvo significantemente asociada a la enfermedad tibioperonea en los hombres, mientras que en las mujeres, la mayor correlación con esa enfermedad distal fue la elevada presión sanguínea sistólica. El riesgo relativo de mortalidad fue de 2 a 7 veces mayor en hombres y mujeres con enfermedad uni y multisegmental, involucrando los segmentos aortoilíacos y femoropoplíteos; la presencia de la enfermedad tibioperonea no aumentó significantemente la mortalidad relativa. Estos resultados sugieren que la etiología y el riesgo de mortalidad asociados con aterosclerosis en las extremidades inferiores puede variar de acuerdo con el sitio anatómico y/o la gravedad de la lesión.

\section{Factores de riesgo}

Los exámenes preventivos periódicos pueden ayudar en la detección oportuna de los problemas del pie. El deterioro de la agudeza visual y la ausencia de sensaciones, con frecuencia son resultado del exceso de confianza que depositan los pacientes en el profesional de la salud para que las detecten. Las opciones para el tratamiento del pie neuropático e isquémico varían en complejidad. La evaluación adecuada y la identificación oportuna de los signos clínicos de úlceras neuropáticas e isquémicas asegurará un buen pronóstico y la mejor intervención para el tratamiento del pie diabético. $^{30}$

Durante el seguimiento prospectivo para documentar el desarrollo de la insensibilidad del pie, Adler y colaboradores ${ }^{31}$ identificaron factores de riesgo de neuropatía sensorial periférica en las extremidades inferiores de diabéticos. El 50\% tenía neuropatía al comenzar el estudio, y sólo $20 \%$ de los que completaron el seguimiento desarrollaron neuropatía. Los factores de riesgo para la neuropatía incidente incluyeron el nivel de glucohemoglobina, historia de úlceras, tabaquismo y nivel de aluminio. El control inadecuado de la glucemia, así como la estatura y la edad también fueron factores de riesgo de neuropatía y se tomaron en cuenta para programar intervenciones. Por último, también se ha observado que la proporción de neuropatías entre los veteranos diabéticos empeora con la ingestión de alcohol. ${ }^{32}$

Missouris y colaboradores ${ }^{33}$ estudiaron la prevalencia, la gravedad, los factores de riesgo vascular y las implicaciones clínicas de la estenosis de la arteria renal en pacientes con enfermedad vascular periférica (EVP). Se encontró una relación positiva significativa entre la presencia de dicha estenosis y la gravedad de la enfermedad vascular periférica. El riesgo de estenosis de la arteria renal fue cuatro veces mayor en los que presentaron tres o cuatro vasos afectados y siete veces mayor en los que tenían cinco o más vasos afectados, en comparación con los que presentaron enfermedad vascular periférica moderada (uno o dos vasos afectados). Además, se concluyó que:

- La presencia de la estenosis de la arteria renal aumentó con la gravedad de la EVP. 
- El riesgo postoperatorio de la revascularización para la EVP parece aumentar en pacientes con estenosis de la arterial renal.

- Para obtener la imagen de las arterias renales por medio de angiografía de sustracción digital con el fin de detectar la enfermedad vascular periférica, los pacientes en estudio deben tener flujo adecuado en la aorta.

Presion plantar

Stess y colaboradores ${ }^{34}$ investigaron el significado de la presión plantar para detectar los sitios de ulceración en pacientes con DM. Por medio del analizador de presión plantar EMED-SF midieron el pico de presión normalizada del cuadro máximo de presión (CMP), la presión-tiempo integral (PTI) y la fuerza-tiempo (FTI) en cada uno de los pies. Dentro del grupo de diabéticos con neuropatía periférica y con úlceras plantares, hubo aumentos significativos en los niveles de CMP y PTI, y se observó que la mayor presión se hacía por debajo de la cabeza del cuarto y el quinto metatarsiano. No hubo diferencias estadísticamente significativas en los niveles de FTI entre los grupos de DM sin neuropatía y sin úlceras. Los pacientes neuropáticos muestran un aumento en las presiones dinámicas de la planta del pie, que los coloca en riesgo de ulceración plantar. Instrumentos como el sistema EMED-SF son útiles para detectar sitios posibles de úlceras plantares en áreas de máxima presión.

\section{Lesiones de pie}

En un estudio controlado de 352 pacientes con DM tipo 2, cuyo propósito era reducir los riesgos de patología en las extremidades inferiores por medio de la educación e intervenciones sistémicas, Litzelman y colaboradores ${ }^{35}$ identificaron y cuantificaron los factores fisiológicos de riesgo independientes para las lesiones de pie en pacientes diabéticos. La dermatitis micótica, la piel seca partida, el edema, las uñas "enterradas", la microalbuminuria, la glicemia en ayuno y la hemoglobina $A_{1 c}$ no fueron predictores significativos.

Frykberg, ${ }^{36}$ por su parte, desarrolló un plan para la prevención y el tratamiento de las úlceras y la osteoartropatía, usando un enfoque multidisciplinario, mediante el cual se estudió la etiopatogenia de las lesiones del pie diabético con énfasis en la neuropatía, la isquemia, la infección y las altas presiones. Estos autores resaltaron la importancia de evaluar esos factores de riesgo apropiadamente con el fin de eliminarlos o disminuirlos considerablemente.

\section{Tratamiento}

La historia del tratamiento del pie diabético nos refiere a épocas previas al uso de antibióticos (década de los 1930), cuando 50\% de los pacientes diabéticos sufrían inevitablemente de amputaciones. El conocimiento de la fisiopatología de la DM permitió su diagnóstico oportuno y su control con insulina y otros medicamentos. El uso adecuado de antibióticos en casos de úlceras diabéticas infectadas, los procedimientos de cirugía vascular para mejorar la circulación y los cuidados generales del paciente con DM han permitido controlar la infección y mejorar la insuficiencia vascular periférica y las neuropatías del pie diabético. El tratamiento efectivo tiene su base en las estrategias integrales para el cuidado de las heridas, evitar el sobrepeso, llevar un control metabólico óptimo, el uso apropiado de antibióticos y, eventualmente, la intervención quirúrgica.

\section{Ulceras}

Se estudiaron los estándares de evaluación y el tratamiento de las úlceras infectadas del pie diabético. ${ }^{37} \mathrm{Los}$ resultados resaltan la necesidad de exámenes sistemáticos y detallados de las extremidades inferiores en cada uno de los pacientes diabéticos admitidos en un hospital, particularmente en aquellos que ingresan con diagnóstico que involucra complicaciones en el pie (amputados y con debridación intraoperativa). No debe omitirse el registro del pulso pedio, la sensación protectora, la evaluación de las estructuras de sostén y las radiografías, además de los cultivos del material de las heridas y de la sangre del paciente. Estas medidas ayudan a la mejor orientación del tratamiento.

\section{Infecciones}

Con frecuencia, las infecciones producidas por Staphylococcus aureus resistente a la meticilina representan un desafío para los médicos que tratan pacientes con heridas del pie; la mayoría de estas infecciones son de origen nosocomial. El riesgo de que se presenten complicaciones del pie puede aumentar con la hospitalización repetida o prolongada y con la presencia de heridas abiertas que facilitan la exposición a la infección. ${ }^{38}$ Los antibióticos deben prescribirse de acuerdo con la susceptibilidad demostrada de las especies involucradas.

Muller y colaboradores ${ }^{39}$ evaluaron la eficacia de la atención en el sistema centralizado de diabetes en la 
entonces Alemania oriental, examinando los parámetros más relevantes de 190 diabéticos tratados con dosis fija de insulina y dieta (cuadro II).

\section{Evaluacion pre y postoperatoria de la isquemia}

Arnold y colaboradores ${ }^{40}$ midieron el índice tobillobraquial (ITB) pre y postoperatorio, el índice de perfusión regional (IPR= tensión del oxígeno transcutáneo pie/tórax $\left[\mathrm{TcpO}_{2}\right]$ ) y la variación en IPR con la elevación de la pierna, en 22 extremidades inferiores isquémicas de 20 pacientes. De acuerdo con sus características, todas las piernas estudiadas se agruparon conforme a la gravedad de la isquemia clínica en presencia o ausencia de DM. Los índices resultantes se compararon usando la prueba t. Los valores aumentaron en el preoperatorio y en la postrevascularización en el ITB (rango 0.27-0.48 y 0.40- 0.54, respectivamente); y en el IPR (rango 0.18-0.45 y 0.48-0.60, respectivamente). Los índices disminuyeron cuando la elevación presentó un rango de 0.07-0.11 y 0.11-0.23, respectivamente. Los valores de ITB e IPR fueron igualmente efectivos en la evaluación preoperatoria de la isquemia clínica y en la postoperatoria durante la perfusión incrementada, independientemente del grado de isquemia o de DM. Durante la elevación todas las piernas exhibieron mayor disminución del flujo sanguíneo a la piel en el periodo postoperatorio que en el preoperatorio, lo que se estimó por medio del IPR. No obstante que disminuyó en el IPR, el postoperatorio de posición fue mayor en los diabéticos que en los no diabéticos $(0.23+/ 0.12$ vs $0.12+$ / 0.06; $p<0.05)$; ello sugiere que la elevación postoperatoria de las piernas diabéticas con lesiones isquémicas de la piel puede dar resultados imprevisibles.

\section{Procedimientos vasculares}

Durante un periodo de más de 17 años, Sayers y colaboradores ${ }^{41}$ acumularon 2930 intervenciones vasculares para el tratamiento de enfermedad oclusiva crónica de la pierna baja. En la mayoría de los casos se trataba de intervenciones para la reconstrucción arterial y la angioplastía percutánea transluminal. Aunque las tasas de amputación mayor de la pierna baja y de mortalidad no se modificaron significativamene, con las intervenciones vasculares hubo otros cambios importantes: a) disminución en la proporción de intervenciones arriba y abajo de la rodilla; b) aumento en el porcentaje de personas tratadas mayores de 75 años, y c) aumento en el número de intentos de revascularización antes de la amputación. La permanencia en el hospital aumentó significativamente para

\section{Cuadro II \\ Porcentaje de diabéticos tratados en el sistema de atención en la antigua Alemania oriental, 1989}

\begin{tabular}{lll} 
Pacientes diabéticos $(\mathrm{n}=190)$ & DM tipo 1 & DM tipo 2 \\
Examinados & $131(69 \%)$ & $59(31 \%)$ \\
\hline Hg glicosilada (normal 4.15\%) & Media $6.3 \%$ & Media $7.4 \%$ \\
\hline Con retinopatía & $35 \%$ & $23 \%$ \\
\hline N efropatía (albuminuria $>20 \mathrm{mg} / \mathrm{L})$ & $29 \%$ & $47 \%$ \\
\hline Hipertensión arterial & $31 \%$ & $69 \%$
\end{tabular}

N ota: $2.1 \%$ del grupo evaluado presentó úlceras de los pies y amputaciones de la pierna baja, y la incidencia anual de hipoglicemia grave fue de 0.07 por paciente. Este estudio mostró que el sistema de atención a la diabetes es efectivo para el control de los pacientes con DM

Fuente: referencia 39

los pacientes a los que se les practicó la amputación y disminuyó significativamente para aquellos a los que se les practicó reconstrucción arterial.

\section{Puentes poplíteo y poplíteo-crural}

La arteria poplítea distal y los vasos crurales proximales representan la localización común de la enfermedad arterosclerótica oclusiva, particularmente en personas con DM. Síntomas como dolor en reposo, ulceraciones distales y gangrena están asociados al alto riesgo de perder la pierna, a menos que se restaure el flujo arterial. La revascularización quirúrgica es el tratamiento más apropiado en estos pacientes, usando la vena autógena para hacer el puente desde la arteria poplítea a la arteria crural por atrás de la estenosis.

El abordaje posterior es un método ideal para conseguir la restauración. Esta técnica usa la misma incisión para exponer el influjo y reflujo de los vasos, minimiza la longitud del conducto necesario, preserva el uso subsecuente de la gran vena safena, reduce la frecuencia de problemas asociados a las incisiones para extraer la vena media y previene problemas de cicatrización. El conocimiento de la anatomía topográfica permite usar el abordaje posterior en pacientes seleccionados con enfermedad oclusiva poplítea e infrapoplítea, lo que a su vez proporciona un instrumento más para el tratamiento de pacientes con riego de perder la pierna por la isquemia. ${ }^{42}$

\section{Gangrena}

Por definición, no hay tratamiento. La gangrena o necrosis se define como muerte local en tejidos blandos vivos. Se puede prevenir y, una vez establecida, las intervenciones locales, sistémicas o de amputa- 
ción dependerán del tipo de infección y del grado de destrucción de los tejidos. En pacientes con DM tipo 2 tratados sin éxito con insulina se ha demostrado por radiografía formación de gas en el tejido blando del pie. Los enfermos con diagnóstico de gangrena gaseosa se tratan con antibióticos y oxígeno hiperbárico. No obstante esa quimioprofilaxis, la gangrena puede progresar y es necesario amputar la pierna. Se han registrado casos de gangrena gaseosa no debida a Chlostridium, en los cuales se aisló Peptstreptococcus del área gangrenosa. El tratamiento con oxígeno hiperbárico es ineficaz en el caso de gangrena gaseosa no producida por clostridios en pacientes diabéticos. ${ }^{43}$

\section{Pronóstico}

Se tiene poca información sobre el destino a largo plazo de pacientes con isquemia crónica de la pierna baja. Dawson y colaboradores ${ }^{44}$ investigaron el riesgo a largo plazo de la isquemia primaria y de hemorragia y embolia cerebrales en pacientes con terapia anticoagulante (derivados de coumarin seguidos de protrombina), después de la reconstrucción de la isquemia crónica de la pierna. El 10\% de los pacientes mostró embolias (isquémicas y hemorrágicas), y $56 \%$ murió. El riesgo y el porcentaje observado a los 5 y 15 años, así como el predictor independiente y el riesgo relativo correspondiente se exponen en el cuadro III.

\section{Sobrevivencia y mortalidad}

Se analizó la sobrevivencia y la mortalidad en 248 casos de amputación de la pierna baja por enfermedad vascular, y después de su rehabilitación con prótesis. ${ }^{45}$ La sobrevivencia promedio fue de 3.5 años, y menor entre las mujeres que entre los hombres. La sobrevivencia también fue menor entre los que tenían enfer- medad vascular periférica en comparación con los diabéticos y entre aquellos que tenían amputación arriba de la rodilla en relación con los que la tenían por debajo de la misma. ${ }^{46}$

\section{Hospitalización}

Bouter y colaboradores ${ }^{47}$ analizaron el impacto del pie diabético en la hospitalización de pacientes con DM en Holanda. En 1988, 20.4\% de todos los diabéticos hospitalizados tenían problemas en el pie. La edad promedio de la población estudiada era de 71.3 años. En pacientes con amputación parcial de la pierna la razón $\mathrm{M} / \mathrm{F}$ fue mayor que en pacientes sin amputación para todos los grupos estudiados. La duración promedio de hospitalización fue de 40.0 días en 1988 y 38.3 días en 1989. Los enfermos con piernas amputadas permanecieron significantemente mayor tiempo en el hospital que los pacientes sin amputación. La letalidad fue de $10 \%$. La mortalidad fue mayor entre pacientes en el estadío Wagner 4 y 5; entre los enfermos en estadío Wagner 1 y 2 ésta fue mayor que en pacientes con osteomielitis. ${ }^{48}$ Aproximadamente $10 \%$ de todos los pacientes fueron enviados a un centro de rehabilitación o a una casa de salud. Tanto la edad promedio de los pacientes hospitalizados como el total del costo de la atención son mayores que lo notificado en estudios previos hechos en EUA. El pie diabético ha impuesto un gran reto a las instituciones encargadas del cuidado de la salud de los gerontes diabéticos, por lo que deben asignarse recursos financieros dirigidos hacia la atención médica preventiva.

También en Holanda ${ }^{49}$ se diseñó un cuestionario dirigido a personal de salud para documentar el tratamiento de las infecciones del pie en pacientes con DM y se implementaron guías de consenso. En los cuestionarios devueltos se registró que la razón principal

\section{TIPO dE RIESGO, PORCENTAJE OBSERVADO, PREDICTOR INDEPENDIENTE Y RIESGO RELATIVO DE EMBOLIA O MUERTE EN ENFERMOS DIABÉTICOS}

\begin{tabular}{|c|c|c|c|c|c|}
\hline Riesgo & 5 años & 15años & Predictor independiente & Riesgo relativo & $p$ \\
\hline Embolia isquémica acumulada & $5 \%$ & $12 \%$ & Infarto de miocardio previo & 3.1 & 0.005 \\
\hline \multirow[t]{2}{*}{ Embolia hemorrágica acumulada } & $3 \%$ & $17 \%$ & Hipertensión sistólica & 4.8 & 0.01 \\
\hline & & & DM tipo 1 & 5.4 & 0.01 \\
\hline \multirow[t]{3}{*}{ Evento vascular mayor } & $29 \%$ & $56 \%$ & Edad avanzada & 1.4 & 0.005 \\
\hline & & & DM tipo 1 & 2.2 & 0.005 \\
\hline & & & Infarto de miocardio previo & 1.8 & 0.01 \\
\hline
\end{tabular}


de hospitalización era el peligro de perder la pierna $(90 \%)$. La mayoría de los pacientes presentaba infección en herida superficial tratada por medio de procedimientos quirúrgicos (90\%); sin embargo, 16\% de los que respondieron consideraron indicada la hospitalización. Si la osteomielitis hubiera estado presente, $81 \%$ de los que respondieron habrían hospitalizado al paciente. El tratamiento con antibióticos fue administrado por el 93\%. En total se usaron 11 antibióticos diferentes. La combinación usada más frecuentemente fue penicilina y lincomicina, y la combinación menos recomendada fue aminoglicósido y lincomicina. La razón para usar una combinación de antibióticos fue la presencia de flora polimicrobiana (93\%). Este estudio indica que el tratamiento del pie diabético es todavía muy diverso a pesar de las guías de consenso.

\section{Conclusiones y recomendaciones}

La intervención, el desarrollo y la evaluación de programas de salud para la prevención, el control y la vigilancia de la enfermedad cardiovascular, la enfermedad vascular periférica, la diabetes mellitus y el pie diabético demandan programas de adiestramiento para médicos, enfermeras, técnicos, gerentes y estudiantes. Para construir programas permanentes se recomienda lo siguiente:

- Desarrollar estrategias que definan metas con indicadores precisos y recursos para la implementación de programas efectivos de salud pública que contribuyan a la prevención, el control y la vigilancia de la DM.

- Mejorar la calidad del diagnóstico y de la atención; promover la mejoría en los estilos de vida y la nutrición; prevenir las enfermedades vasculares y disminuir la morbilidad, las complicaciones y la mortalidad producidas por la DM, en coordinación con otros programas de promoción y protección de la salud.

- Incorporar el programa en modelos de atención integrada.

- Asegurar la disponibilidad de tecnologías apropiadas para el diagnóstico de la DM y sus complicaciones.

- Garantizar el abastecimiento de insulina y otros medicamentos, especialmente en los sistemas locales de salud.

- Difundir el conocimiento y fomentar la capacidad resolutiva entre las personas afectadas por la DM.

- Organizar servicios efectivos para el manejo de las estenosis en las arterias de las extremidades pélvicas.
- Prácticar una evaluación simple y regular de la sensación de la pierna baja en personas con diabetes y proveer de equipo protector y asesoría a aquellas en riesgo de presentar úlceras en el pie.

\section{Referencias}

1. O rganización Panamericana de la Salud/O rganización Mundial de la Salud. 39a. Reunión del Consejo Directivo O PS/O MS. W ashington, D.C.: O PS/O MS, 1996.

2. Pan A merican Health O rganization/W orld Health O rganization. C elebrating 95 years: 1902-1997-Protecting A mericas' health. Diabetes cases in the Americas expected to jump from 30 million to 45 million.W ashington, DC.: PAHO/W HO, 1997.

3.W orld Health O rganization. Control of hereditary diseases. Report of a W HO Scientific Group. Ginebra:W HO Techn Rep Ser, no. 865,1996.

4. World Health 0 rganization. Investing in health research and development. Ginebra: Report of the Ad Hoc Committee on Health Research Relating to Future Intervention O ptions, 1996.

5. Jelinek JE. The skin in diabetes. Philadelphia: Leo \& Febiger, 1986.

6. Esco bedo-De la Peña J, Santos-Burgoa C. La diabetes mellitus y la transición de la atención a la salud. Salud Publica Mex 1995;37(1):37-45.

7. 0 rganización Mundial de la Salud. Prevención de la diabetes mellitus. Ginebra: Informe de un Grupo de Estudio de la OMS. Ser Inf Tecn, no. 844, 1994: 81.

8. McInnes A. Know how-Diabetic foot ulceration. N urs Times 1997; 93(28):34-35.

9. Arora S, LoGerfo FW. Lower extremity macrovascular disease in diabetes. Am Podiatr Med Assoc 1997;87(7):327-331.

10. Report of the Expert Committee on the Diagnosis and Classification of Diabetes Meliitus. Diabetes Care 1997;20:1183-1197.

11. Eastman RC,Vinicor F. Science: moving us in the right direction. Diabetes Care 1997;20:1057-1058.

12. McC ance DR, Hanson RL, Pettit DJ, Bennett PH, Hadden DR, Knowler W C. Diagnosing diabetes mellitus-D o we need new criteria? Diabetologia 1997; 40:247-255.

13. D avidoff F. Blood sugar, disease, and nondisease.Ann Intern Med 1997; 127(3): 235-237

14. 0 rganización Mundial de la Salud. Factores de riesgo de enfermedades cardiovasculares: nuevas esferas de investigación. Ginebra: Informe de un Grupo Científico de la O MS. Ser Inf Tecn, no. 841, 1994.

15.W allace DC. Mitochondrial genetics: A paradigm for aging and degenerative diseases? Science 1992; 256: 628-632.

16. Dorman J. Molecular epidemiology of insulin-dependent diabetes mellitus:W HO Diamond Project. Gac Med Mex: 133 suppl 1:151-154.

17. $O$ lerup 0 , Aldener- $C$ annava $A$, Fogdell- $H$ ahn $A$, G etty RR, W agenknecht DR, Mclntyre JA. DQB1*0202 and the new DQB1*0203 allele: $a$ fourth pair of DQB1 alleles differing only at codon 57.Tissue Antigens 1997:49:271-273.

18. Santos-Martín JL, Pérez-Bravo F, Carrasco E, Icaza G, Calvillan M, Albala C. Different statistical models used in the calculation of the prevalence of insulin-dependent diabetes mellitus according to the polymorphism of the HLA-DQ region. Immunol C ell Biol 1997;75(4):351-355.

19. C acciatori V, D ellera A, Bellavere F, Bongiovanni LG, Teatini F, Gemma $M L$ et al. Comparative assessment of peripheral sympathetic function by postural vasoconstriction arteriolar reflex and sympathetic skin response in N IDDM patients. Am J Med 1997;102(4):365-370.

20. Ubbink DT, Kitslaar PJ, Tordoir JH, Reneman RS, Jacobs MJ. Skin microcirculation in diabetic and nondiabetic patients at different stages of lower limb ischaemia. Eur J Vasc Surg 1993; 7(6): 656-659. 
21. Caputo GM, Joshi $\mathrm{N}$,W eitekamp MR. Foot infections in patients with diabetes. Am Fam Physician 1997;56(1):195-202.

22. Strandness DE Jr, Carter SA. O utcome criteria in patients with peripheral arterial disease. Ann Vasc Surg 1993; 7(5): 491-496.

23.Thomas PK. Classification, differential diagnosis, and staging of diabetic peripheralneuropathy. Diabetes 1997;46 suppl 2:S54-S57.

24. Craig JG,A min MB, W u K, Eyler W R, Van Holsbeeck MT, Bouffard JA et al. 0 steomyelitis of the diabetic foot: MR imaging-pathologic correlation. Radiology 1997;203(3):849-855.

25. Harvey J, Cohen MM.Technetium-99-labeled leukocytes in diagnosing diabetic osteomyelitis in the foot. J Foot Ankle Surg 1997;36(3):209-214. 26. $N$ ijhof MW, 0 yen W J,Van Kampen A, Claessens RA,Van der Meer JW, Corstens $\mathrm{FH}$. Evaluation of infections of the locomotor system with indium-111-labeled human IgG scintigraphy. J Nucl Med 1997;38(8): 1300-1305.

27. Chen YJ, W ang CL, Lo SK. Cutaneous microcirculation of diabetic foot: Combined isolated cold stress testing and laser Doppler flowmetry study. J Formos Med Assoc 1997;96(8):606-612.

28. De N eeling JN, Beks PJ, Bertelsmann FW, Heine RJ, Bouter LM. Sensory thresholds in older adults: reproducibility and reference values. Muscle Nerve 1994; 17(4): 454-461.

29. Vogt MT, W olfson SK, Kuller LH. Segmental arterial disease in the lower extremities: correlates of disease and relationship to mortality. J Clin Epidemiol 1993; 46(11): 1267-1276.

30. Young T. Management of the diabetic patient: causes of leg ulceration. Br J N urs 1997;6(8):418.

31. Adler AI, Boyko EJ, A hroni JH, Stensel V, Forsberg RC, Smith DG. Risk factors for diabetic peripheral sensory neuropathy. Results of the Seattle Prospective Diabetic Foot Study. Diabetes Care 1997;20(7):1162-1167.

32. Day MR, Harkless LB. Factors associated with pedal ulceration in patients with diabetes mellitus. J Am Podiatr Med Assoc 1997;87(8): 365369.

33. Missouris CG, Buckenham T, Cappuccio FP, MacG regor GA. Renal artery stenosis: A common and important problem in patients with peripheral vascular disease. Am J Med 1994; 96(1): 104.

34. Stess RM, Jensen SR, Mirmiran R. The role of dynamic plantar pressures in diabetic foot ulcers. Diabetes Care 1997;20(5):855-858.

35. Litzelman DK, Marriott DJ,Vinicor F. Independent physiological predictors of foot lesions in patients with NIDDM. Diabetes Care 1997; 20(8):1273-1278.
36. Frykberg RG. Team approach toward lower extremity amputation prevention in diabetes. J Am Podiatr Med Assoc 1997;87(7):305-312.

37. Edelson GW, Armstrong D G , Lavery LA, C aicco G. The acutely infected diabetic foot is not adequately evaluated in an inpatient setting. J Am Podiatr Med Assoc 1997:87(6):260-265.

38. Day MR, Armstrong DG. Factors associated with methicillin resistance in diabetic foot infections. J Foot Ankle Surg 1997;36(4):322-325.

39. Muller UA, Rossl S, Klinger H, Geisenheiner S, Chantelau EA. Q uality of centralized diabetes care: a po pulationbased study in the German D emocratic Republic 1989-1990. Acta Diabetol 1993; 30(3): 166-172.

40. Arnold T, Karabinis V, Sano C, Gensler T, U gaeri H, Samuels L, et al. Revascularized diabetic limbs: Positional changes in regional perfusion index. Am Surg 1993; 59(11):746-749.

41. Sayers RD,Thompson M M, Varty K, Jagger C, Bell PR. C hanging trends in the management of lowerlimb ischaemia: a 17 year review. $B r$ J Surg 1993:80(10):1269-1273.

42. 0 uriel K. Posterior exposure for popliteal-crural bypass: a useful approach. Semin Vasc Surg 1997;10(1):23-30.

43. O wada R, Kanamori A, Hirai N, Yajima Y. [A case of N IDDM with nonclostridial gasproducing infection in the lower limbthe effects of hyperbaric oxygen therapy]. Kansenshogaku Zasshi 1994; 68(2): 263-267. 44. Dawson I, Van Bockel JH, Ferrari MD, Van der Meer FJ; Brand R, Terpstra JL. Ischemic and hemorrhagic stroke in patients on oral anticoagulants after reconstruction for chronic lower limb ischemia. Stroke 1993; 24(11): 1655-1663.

45. Gaspar E, Pillar T, Dickstein R. [Survival and mortality in amputees with dysvascular lower limbs]. Harefuah 1993;125(11): 393-395.

46. Ewins DL, Bakker K, Young MJ, Boulton AJ. Alternative medicine: potential dangers for the diabetic foot. Diabet Med 1993; 10(10): 980-982. 47. Bouter KP, Storm AJ, De Groot RR, U itslager R, Erkelens DW, Diepersloot RJ.The diabetic foot in Dutch hospitals: Epidemiological features and clinical outcome. Eur J Med 1993; 2(4): 215-218.

48. W agner FW Jr. The diabetic foot. 0 rthopedics 1987;10(1):163-172. 49. Storm A], Bouter KP, D iepersloot R], Erkelens DW. [Large variation in the treatment of foot infections in diabetic patients in The $N$ etherlands]. N ed Tijdschr Geneeskd 1994; 138(11): 569-573. 